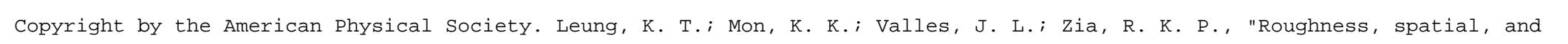

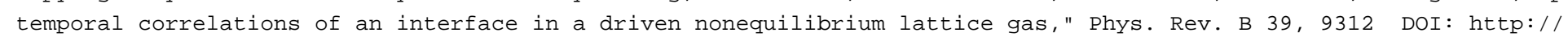
dx.doi.org/10.1103/PhysRevB.39.9312

PHYSICAL REVIEW B

VOLUME 39, NUMBER 13

1 MAY 1989

\title{
Roughness, spatial, and temporal correlations of an interface in a driven nonequilibrium lattice gas
}

\author{
K.-t. Leung* and K. K. Mon \\ Department of Physics and Astronomy and Center for Simulation Physics, University of Georgia, Athens, Georgia 30602 \\ J. L. Vallés ${ }^{\dagger}$ \\ Courant Institute of Mathematical Sciences, New York University, New York 10012 \\ R. K. P. Zia \\ Center for Transport Theory and Mathematical Physics, and Physics Department, Virginia Polytechnic Institute \\ and State University, Blacksburg, Virginia 24061
}

(Received 1 December 1988)

\begin{abstract}
The interface of a stochastic Ising lattice gas driven into a nonequilibrium steady state by a constant, uniform electric field $E$ parallel to the interface is studied by extensive Monte Carlo simulation in two dimensions. Dependence on the system size and the field strength of the interface profile, roughness, time, and spatial correlation functions and structure factors are found numerically, by means of a coarse-graining method. The interface at zero field is shown to be rough by the divergence of both the interface width and correlation time. As soon as $E$ is turned on, the interface becomes smooth. We argue that the general results may be extended to other similar nonequilibrium systems, and in higher dimensions.
\end{abstract}

\section{INTRODUCTION}

The physics of interfaces has received considerable attention in recent years. ${ }^{1}$ It encompasses a wide range of low-temperature physical phenomena of fundamental importance. One subject that has been extensively studied has to do with the roughening transition, ${ }^{2}$ which is believed to occur in bulk dimension $d=3$ at a finite roughening transition temperature $\left(T_{R}\right)$ below the critical temperature $\left(T_{c}\right)$. On approaching $T_{R}$ from below, the interface separating two coexisting phases becomes rough as the interface width diverges in the thermodynamic limit. In the rough phase, the correlation length diverges along the interface, leading to a power-law decay of the two-point correlation function. In contrast, the interface for two bulk dimensions $(d=2)$ is rough for all temperatures $T<T_{c}$, i.e., $T_{R}=0$. It has been shown that some two-dimensional solid-on-solid (SOS) models ${ }^{3}$ (which describe an interface in three bulk dimensions without bubbles and overhang excitations) undergo, at a finite $T_{R}$, a roughening transition whose critical properties are of the Kosterlitz-Thouless type. ${ }^{4}$ The $d=3$ Ising-model interface is believed to exhibit a similar transition of the same universality class ${ }^{5}$ at a slightly lower $T_{R}$. Since the singularities predicted at $T_{R}$ are very weak, they are difficult to detect experimentally. However, roughening on crystal surfaces seems to have been observed in diffraction experiments. ${ }^{6}$

The roughening transition is usually destroyed by the application of external fields (customarily termed "pinning fields") such as gravity in fluids or a magnetic field gradient in magnetic systems. ${ }^{7}$ In real materials, this transition is often masked by the presence of impurities and defects. The interface is thus inevitably influenced to some extent by external fields in an uncontrolled way. A similar, but physically different, situation is when the field is controlled to maintain the system in a steady state away from equilibrium. A question of apparent physical interest is whether or not such a field is relevant to the roughening transition. This is not at all clear at the outset. In this paper we attempt to answer this question within the context of a recently studied system. In its simplest form, it is represented by an Ising lattice gas of charged particles driven by an external, constant, uniform electric field $(E){ }^{8}$ For bulk properties of this model, progress has emerged mainly from computer simulations. This is due to the inherent difficulties of formulating analytically statistical mechanical treatments for nonequilibrium systems. Such treatments necessarily start from the equation of motion, e.g., the master equation, ${ }^{9}$ or equivalently a dynamic functional. ${ }^{10}$ From simulations in both $d=2$ and $d=3$, the systems were observed to phase separate below a certain critical temperature which is higher than the zero-field (Onsager) value. Such separations are very anisotropic, with the interface parallel to the field direction under periodic boundary conditions. The transition is believed to be of second order, as concluded from both theories and simulations (at critical density). However, the nature of the transition is still controversial, since theories predict mean-field behavior, ${ }^{9,10}$ disagreeing with available simulation results. 8,11 Below criticality, there are studies of linear stabilities of the interface based on a phenomenological theory. ${ }^{12}$

We found, by computer simulation, that the field destroys interface roughness for a wide range of values of 
$E .^{13}$ Though we study only a $d=2$ system, the results have implications in $d=3$. This paper is organized as follows. Section II describes the simulation method for the interface. The main results are stated in Sec. III. Section IV contains discussions and concluding remarks.

\section{SIMULATION METHOD}

Using standard Monte Carlo methods ${ }^{14}$ on the twodimensional Ising model with spin-exchange dynamics, we mimic the motion of particles with number (charge) conservation. We will use spin or particle language interchangeably, with spin $\sigma=+1(-1)$ at each site of a square lattice representing a particle (hole). The spins inter- act via the usual ferromagnetic Ising Hamiltonian $H=-J \sum_{n n} \sigma_{i} \sigma_{j}$, where $J>0$, and the sum is over nearest-neighbor pairs. The external field $E$, chosen to be in the $+x$ direction, enters through the (Metropolis) jump rates so that the relative probabilities of particles jumping along (against) $E$ are enhanced (suppressed). ${ }^{8}$ Throughout this paper, temperature $T$ is measured in units of the zero-field critical temperature $T_{c} \approx 2.269,{ }^{15}$ while $E$ is expressed in units of $J$. The size of the system is $L_{x} L_{y}$ with a fixed number of particles $N \equiv L_{x} L_{y} / 2$. Below the critical temperature $T_{c}(E)$, the system phase separates into particle-rich and particle-poor phases. The interface between these two phases is forced to lie (on the average) along $y=L_{y} / 2$, by placing only particles and holes on the row at $y=0$ and $L_{y}+1$, respectively. Periodic boundary condition (PBC) is imposed in the $x$ direction (along $E$ ).

To obtain a sensible description of the interface, we need to consider the interplay between various lengths in the system. ${ }^{16}$ Our main concern is to examine the size $\left(L_{x}\right)$ dependence of the width $(w)$ of the interface at temperature below $T_{c}$. There are two contributions to $w$ : One comes from the intrinsic width of the interface, which is of the order of the bulk correlation length $\xi_{B}$, and another comes from thermal fluctuations of the local mean position of the interface (capillary waves). The divergence of the former as the bulk critical point is approached is responsible for the disappearance of the interface, while the latter gives rise to the interface roughness. At low temperature (which we ensure in our simulation), the latter contribution dominates. Instead of measuring the interface width by computing moments of the density profile alone, ${ }^{17}$ we define interface configurations by associating a local height function $h(x)$ with each bulk configuration. This enables us to calculate height-height correlation functions. As far as we are aware, this is the first such calculation using Monte Carlo simulation on a bulk model. To arrive at $h(x)$, we first coarse grain the bulk density of each configuration so as to obtain a smooth density. Our method consists of spreading the "raw" particle density ( 0 or 1$)$ at each site to its four neighbors by assigning 0 or $\frac{1}{5}$ to all five sites. When iterated, bubbles and overhangs disappear. With such a smooth bulk density, it is possible to define a single-valued function $h(x)$. This method has the advantage over the one using the density profile of suppressing the influence of bulk excitations (in the form of bubbles) on the interface. While the physics should be independent of the details of how we coarse grain, this also presents a natural way to study local properties of the interface.

Note that the coarse graining is performed on a copy of the particle configuration after every fixed number of updates [ 25 or 50 in units of Monte Carlo step per site (MCS)]. The evolution of the interface is still given by the bulk evolution. The size $L_{y}$ normal to the interface does not play an important role here, provided that it is large enough to avoid repulsion between the interface and the boundaries.

\section{SIMULATION RESULTS}

We perform large-scale Monte Carlo simulations on a wide range of system sizes $\left(L_{x}\right.$ from 4 to $60, L_{y}$ from 24 to 36 ) and field values ( $E$ from 0 to 50 , which is essentially infinity). We choose temperatures $T=0.75$ for $E=0$, 0.5 , and $T=0.9$ for larger $E$. The relaxation time is extraordinarily long, especially for $E=0$. The origin of this difficulty is well known: Particle number is conserved locally. Particles must be transported both into the bulk and along the interface in order to change an interface configuration. Mathematically, this property is manifested in nonlocal (both spatial and temporal) couplings between different parts of the interface. ${ }^{18}$ Therefore, very long runs $\left(0.5\right.$ to $\left.2 \times 10^{6} \mathrm{MCS}\right)$ are required to generate statistically meaningful data for small $E$. Long relaxation time is evident from the following time-correlation measurement.

The calculations reported here took about $300 \mathrm{~h}$ of CPU time on the Cyber 205. Errors are estimated from the spread of data from three to four different runs, as well as from standard deviations in time averagings. The results are following.

\section{A. Density profile $m(y)$}

The density profile is given by the mean magnetization, in spin language, along each row,

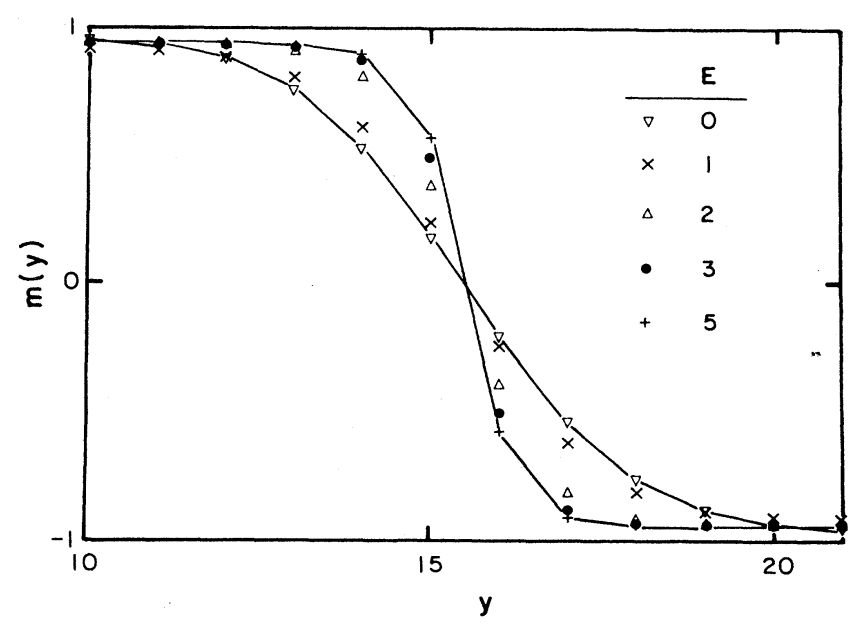

FIG. 1. The density profile obtained by averaging $\sigma(x, y)$ over $x$. Temperatures are $T=0.75$ for $E=0$, and 0.9 for $E \neq 0$. $L_{x}=30$. 


$$
m(y)=\left(1 / L_{x}\right) \sum_{x} \sigma(x, y) .
$$

We ensure that the interface is free from boundary effects by choosing $L_{y}$ to be much larger than both the interface width and the lengths (of order $\xi_{B}$ ) characterizing the decay from the $m= \pm 1$ boundary values to the mean values in the bulk phases. It is obvious from Fig. 1 that the interface becomes much sharper at larger field $E$.

\section{B. Width of interface $w$}

Many possible measures of the width are conceivable. ${ }^{19}$ One natural choice is given by the root mean square of the height variable $h(x)$,

$$
w^{2}=\left\langle(h-\langle h\rangle)^{2}\right\rangle \text {. }
$$

Alternatively, we consider the height-height correlation function

$$
C(x)=\left\langle[h(x)-h(0)]^{2}\right\rangle,
$$

and define $2 w^{2}$ as $C_{\max }$, the maximum value of $C(x)$. In a finite system under periodic boundary conditions, $C_{\max }=C\left(L_{x} / 2\right)$. We also calculate the second moment of the gradient of the density profile $m(y)$. All three measures give similar and consistent results. It turns out that the results from $m(y)$ have the largest statistical errors, which is believed to arise from the bulk excitations.

A plot of $w^{2}$ as a function of $L_{x}$ for various values of $E$ has been published previously (Fig. 1 of Ref. 13). The increase of $w^{2}$ towards its asymptotic value is slower for smaller $E$. The present level of accuracy of the data with small field $(E=0.5)$ does not support a definitive conclusion, although we believe that the interface will be asymptotically rough only at $E=0$. For $E=0, w^{2}$ appears to diverge linearly in $L_{x}$ as $L_{x} \rightarrow \infty$, as predicted by exact calculations, ${ }^{20}$ capillary wave theory, ${ }^{21}$ and as confirmed by molecular dynamics simulation. ${ }^{22}$ For $E>0$, by analyzing the finite-size behavior of $w^{2}$, we found that the best fit shows $w^{2}$ saturating at a finite

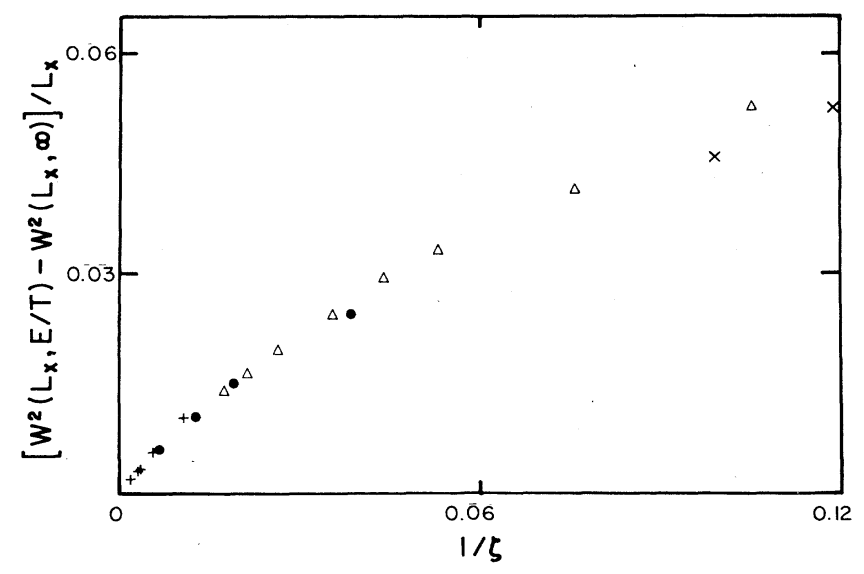

FIG. 2. A scaling plot of the width in the limit of strong $E$ and large $L_{x} ; \zeta \equiv L_{x}(E / T)^{2.5}$. All symbols have the same meaning as in Fig. 1. value as $L_{x}$ increases. Thus, we conclude that the interface is smooth. The reader is referred to Ref. 13 for details of the analysis.

In the large- $L_{x}$ limit, we expect $w^{2}$ to diverge as $E \rightarrow 0$. We will return to this small- $E$ limit later. At the opposite limit of large $E$, we probe the way in which the width approaches the finite value at $E=\infty$ by plotting

$$
\left[w^{2}\left(L_{x}, E, T\right)-w^{2}\left(L_{x}, 50, T\right)\right] / L_{x}
$$

against $1 / \zeta$, where $\zeta \equiv L_{x}(E / T)^{s}$ and $s$ is an adjustable exponent. We found that the data scale, for large $\zeta$ with $s \approx 2.5$ (see Fig. 2), as they collapse on a single curve. This scaling implies for the large- $E$ limit

$$
w^{2}\left(L_{x}, E, T\right)-w^{2}\left(L_{x}, 50, T\right) \approx L_{x} X(\zeta)
$$

with the asymptotic behavior $X(\xi) \sim 1 / \xi$ as $\xi \rightarrow \infty$. We interpret this behavior as the existence of a new length scale $\lambda_{E}$ provided by $E$, scaling as $E^{-s}$ as $E \rightarrow \infty$. Though this scaling may be approximate, it is distinct from critical scaling. ${ }^{23}$

\section{Height-height correlation function $C(x)$}

As for any spatial correlations, periodic boundary conditions introduce serious finite-size effects when the correlation length is comparable to the system size. ${ }^{24}$ This is the case for $E=0$. The linear behavior, expected to hold for all large $x$ for an infinite system, now only extends to within a small fraction of $L_{x}$ from $x=0$, long before $x$ reaches $L_{x} / 2$. The agreement is good between our normalized measured values $C(x) / C_{\max }$ and the prediction of capillary wave theory ${ }^{21}$ (with PBC imposed). For larger $E$, the bendover due to the finite-size effect of PBC is less significant, which is consistent with the reduction of the correlation length (see Fig. 3). For a given $E$, it appears that the interface is rough only for small distances. Beyond some effective crossover length controlled by $E$,

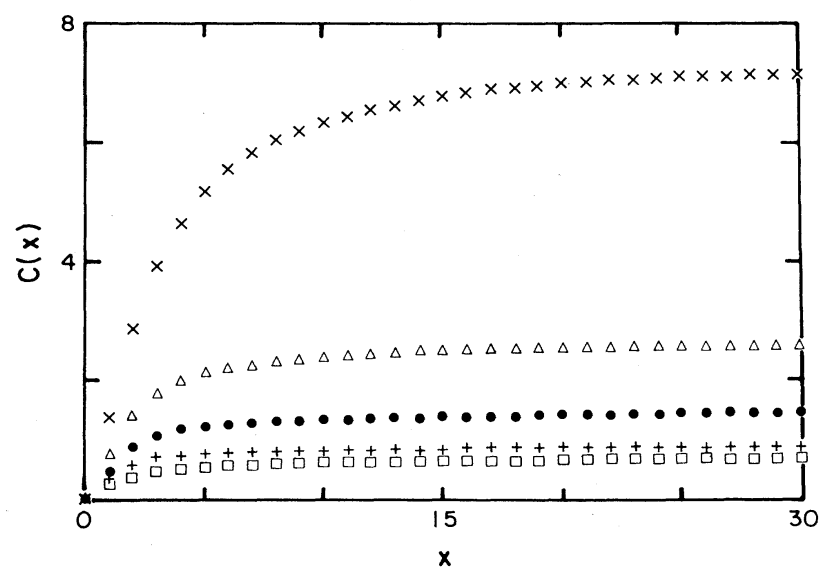

FIG. 3. The height-height correlation function as a function of distance for $L_{x}=60$. For strong $E$, the effect of finite size due to $\mathrm{PBC}$ is negligibly small for $x<20$. The $E=0$ correlation is an order of magnitude off the scale of this graph. All symbols have the same meaning as in Fig. 1, except $\square$ is for $E=50$. 


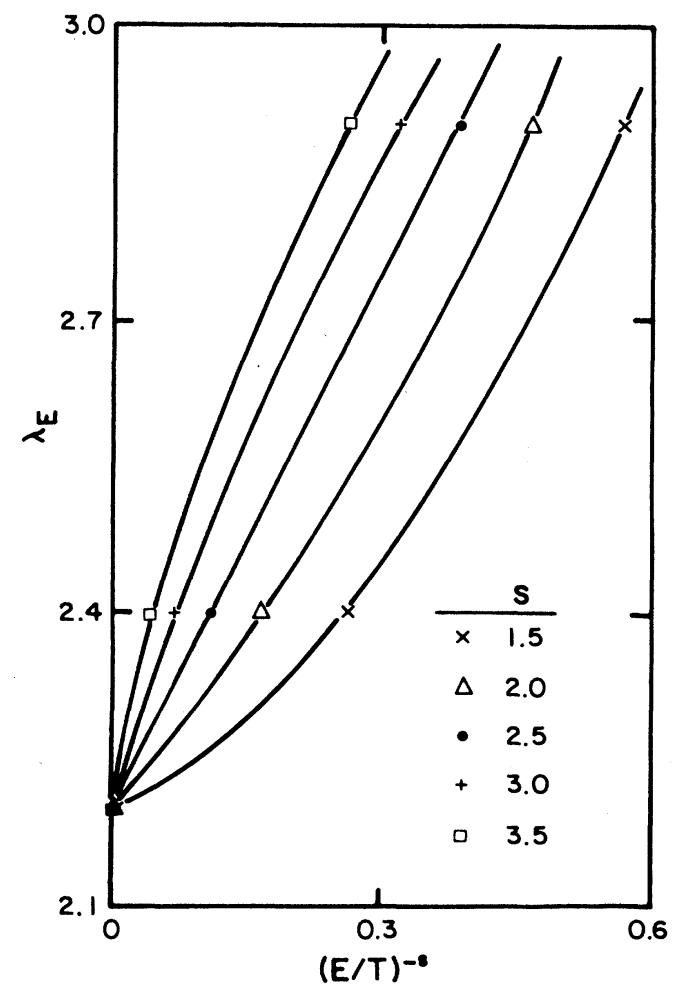

FIG. 4. A plot of an effective crossover length $\lambda_{E}$ vs $(E / T)^{-s}$ for various $s$. The fit is closest to linear for $s \approx 2.5$. $E$ 's here are 3,5 , and 50 .

the interface is smooth, resulting in the plateaus found in the $C(x)$ and $w^{2}$ curves. We get a rough estimate of this length from $C(x)$ by the crossing of the extension of the initial linear behavior and the asymptote at large distance and found approximately $\lambda_{E} \sim E^{-2.5}$ (see Fig. 4). The dependence on $E$ of this length agrees with the above independent estimate in the scaling plot of $w^{2}$.

\section{Structure factor $G(q)$}

The structure factor

$$
G(q) \equiv\langle\delta h(q) \delta h(-q)\rangle
$$

is just the Fourier transform of the height-height correlation function

$$
C(x)=2\left[\left\langle(\delta h)^{2}\right\rangle-\langle\delta h(x) \delta h(0)\rangle\right],
$$

where $\delta h(x) \equiv h(x)-\langle h\rangle$. In a published plot (Fig. 2 of Ref. 13), we observe that $G(q)^{-1}$ increases linearly as $q^{2}$ for $E=0$. This divergence of $G(q)$ at $q=0$ is a manifestation of the Goldstone mode associated with the spontaneous symmetry breaking of translational invariance by the presence of the interface. ${ }^{25}$ For finite $E$, the data deviate from linearity near $q=0$. Recall that the effect of conventional pinning fields is to introduce a mass to the spectrum $^{21,7}$ which forces $G(0)^{-1}$ to be positive. The behavior under $E$ is evidently different. The finite sizes of our systems prevent us from probing further to see if $G(0)<\infty$, or if there is some correction term of the form

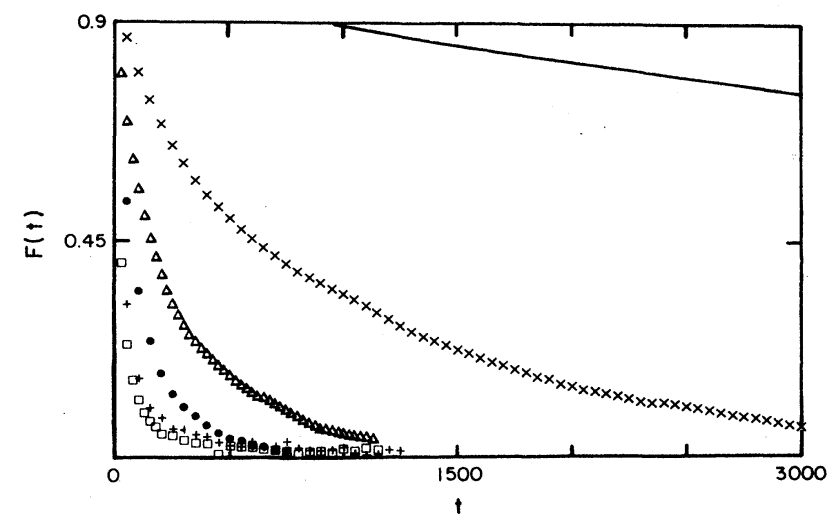

FIG. 5. Time-correlation function of the width for $L_{x}=30$. The rapid decays for large $E$ indicates smooth interface. $t$ is measured in MCS. All symbols have the same meaning as in Fig. 3. Solid curve is for $E=0$.

$q^{\alpha}$ with $\alpha<2$, in a small- $q$ expansion of $G(q)^{-1}$. In either case, the interface is smooth. This interesting issue deserves further study, but is presumably difficult, as one does not know in advance how small $q$ must be before asymptopia sets in. Perhaps a finite-size analysis is again helpful.

\section{E. Time-displaced correlation function $F(t)$}

We measure the following quantity to examine the dynamical aspect of the interface:

$$
F(t) \equiv \frac{\left\langle w^{2}(t) w^{2}(0)\right\rangle-\left\langle w^{2}\right\rangle^{2}}{\left\langle w^{4}\right\rangle-\left\langle w^{2}\right\rangle^{2}}
$$

Figure 5 summarizes the disparity of time scales associated with the interface as $E$ varies. To get some quantitative feeling, we fit $F(t)$ to a sum of two exponential functions, and extract from it an effective time scale $\tau\left(L_{x}, E\right)$ by a weighted average of the two fitted decay constants. On examining the size dependence of $\tau$, we observe that

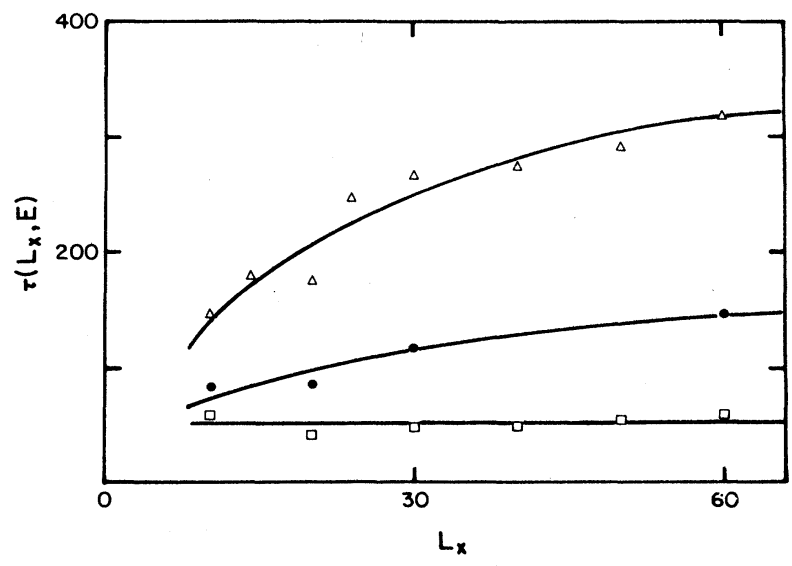

FIG. 6. Effective correlation time, in MCS, for large $E$ as a function of $L_{x}$. Note saturation in the large- $L_{x}$ limit. All symbols have the same meaning as in Fig. 3. 
$\tau\left(L_{x}, E=0\right)$ increases with $L_{x}$ in a way faster than linearly. This is analogous to critical slowing down. ${ }^{26}$ For larger $E$, the increase of $\tau$ with $L_{x}$ is slower, and $\tau$ appears to saturate at large $L_{x}$ similar to the behavior of the width (see Fig. 6). In the infinite $E$ limit, all $F(t)$ for different system sizes practically collapse onto one curve. This provides strong support for the assertion that the interface is smooth, even for moderate $E$. Returning to the case of $E=0$, we are not able to access the long-time tail due to long-relaxation times. Thus $\tau$ for $E=0$ characterizes the relatively short-time decay. It is believed that the long-time decay will be characterized by a correlation time which scales as $L_{x}^{z}$, with $z=3$ according to classical theories. $^{27}$

\section{DISCUSSIONS AND CONCLUDING REMARKS}

We believe that the suppression of interfacial roughness by the external field is a general feature of driven steady-state systems, despite the fact that we have only studied a $d=2$ lattice system in a limited range of temperature. It is reasonable to suggest by analogy with gravity that the results are true for all temperatures below $T_{c}(E)$, although this conjecture is subject to further verification. Intuitively, the microscopic mechanism of "smoothing" should also be applicable in higher dimensions. This mechanism calls for particles to jump along the field much more frequently than normal to it. But it is the latter kind of jumps which ultimately lead to interface roughness. In other words, the time scale associated with the creation of a long-wavelength modulation in the inerface is much longer than that of destruction by a strong field. Thus for a given $E$, the interface appears to be rough at small distances. At distances larger than a certain crossover $\lambda_{E}$, at which the above two time scales become comparable in magnitude, the interfacial width stabilizes. For lattice systems in $d=3$ with a positive $T_{R}$, the interplay between $T$ and $E$ should be very delicate and interesting near $T=T_{R}$ and $E=0$. This problem deserves further study.

For other systems in nonequilibrium steady states, it is conceivable that some share certain common features with the model we studied, though they are mostly more complicated. One of the key characteristics is a constant flux of constituents along the interface. In such systems we speculate that the interface is also smooth.

Beyond these are systems where the flux is orthogonal to the interface. ${ }^{28}$ The question of stability and the possibility of dynamical scaling naturally arise. The present system seems to provide one simple model in which such issues can be investigated in detail, namely, when the field is directed to be normal to the interface. It is interesting to note that if we change the boundary condition along $E$ from $\mathrm{PBC}$ to hard walls, and that normal to $E$ from hard walls to $\mathrm{PBC}$, then $E$ is precisely gravity, and we should also see a smooth interface for any finite $E$, with $G(q) \approx 1 /\left(q^{2}+\right.$ const) for small $q .{ }^{21,7}$

As noted previously, a system with $E=0$ is critical (with infinite correlation length and time). So the "nearcritical" small- $E$ limit is particularly interesting, with $E$ playing the role of a reduced temperature. On physical grounds, the interface should obey scaling, since the interface should be rough on all scales up to the interface correlation length (which diverges as $E \rightarrow 0$ ). We should investigate this limit and understand to what universality class this behavior belongs. As far as its demand on computer resources is concerned, this represents another large-scale project in the future.

\section{ACKNOWLEDGMENTS}

The following sources of support are gratefully acknowledged: the Advanced Computational Methods Center, the Research Foundation of University of Georgia, the Comisió Interdepartmental de Recerca i Innovació Tecnològica (CIRIT) de la Generalitat de Catalunya (Spain), and the Division of Materials Research of the National Science Foundation.
${ }^{*}$ Present address: Physics Department, Virginia Polytechnic Institute and State University, Blacksburg, VA 24061.

$\dagger$ Present address: Physics Department, University of Minnesota, Minneapolis, MN 55455.

${ }^{1}$ For reviews on interfaces, see, e.g., D. Jasnow, Rep. Prog. Phys. 47, 1059 (1984); R. K. P. Zia, in Statistical and Particle Physics: Common Problems and Techniques, edited by K. C. Bowler and A. J. McKane (Scottish Universities Summer School in Physics Press, Edinburgh, 1984), pp. 247-301, and references therein.

${ }^{2}$ For a recent review, see $H$. van Beijeren and I. Nolden, in Topics in Current Physics, edited by W. Schommers and P. von Blanckenhagen (Springer, Berlin, 1987), Vol. 43, pp. 259-300, and references therein.

${ }^{3}$ S. T. Chui and J. D. Weeks, Phys. Rev. B 14, 4978 (1976); H. van Beijeren, Phys. Rev. Lett. 38, 993(1977).

${ }^{4}$ J. M. Kosterlitz and D. J. Thouless, J. Phys. C. 6, 1181 (1973); J. M. Kosterlitz, ibid. 7, 1046 (1974).

${ }^{5}$ K. K. Mon, S. Wansleben, D. P. Landau, and K. Binder, Phys.
Rev. Lett. 60, 708 (1988).

${ }^{6} J$. Villain, D. Grempel, and J. Lapujoulade, J. Phys. F 15, 809 (1985); J. Lapujoulade, J. Perreau, and A. Kara, Surf. Sci. 129, 59 (1983); E. H. Conrad, R. M. Aten, D. S. Kaufman, L. R. Allen, M. den Nijs, and E. K. Riedel, J. Chem. Phys. 84, 1015 (1986); M. den Nijs, E. K. Riedel, E. H. Conrad, and T. Engel, Phys. Rev. Lett. 55, 1689 (1985).

${ }^{7}$ J. D. Weeks, Phys. Rev. Lett. 52, 2160 (1984).

${ }^{8}$ S. Katz, J. L. Lebowitz, and H. Spohn, Phys. Rev. B 28, 1655 (1983); J. Stat. Phys. 34, 497 (1984); 38, 725 (1985).

${ }^{9}$ H. van Beijeren and L. S. Schulman, Phys. Rev. Lett. 53, 806 (1984).

${ }^{10}$ K.-t. Leung and J. L. Cardy, J. Stat. Phys. 44, 567 (1986); H. K. Janssen and B. Schmittmann, Z. Phys. B 64, 503 (1986); K. Gawadzki and A. Kupiainen, Nucl. Phys. B269, 45 (1986).

${ }^{11}$ J. L. Vallés and J. Marro, J. Stat. Phys. 49, 89 (1987).

${ }^{12}$ K.-t. Leung, J. Stat. Phys. 50, 405 (1988); A. HernàndezMachado and D. Jasnow, Phys. Rev. A 37, 656 (1988).

${ }^{13}$ K.-t. Leung, K. K. Mon, J. L. Vallés, and R. K. P. Zia, Phys. 
Rev. Lett. 61, 1744 (1988).

${ }^{14}$ See, e.g., Monte Carlo Methods in Statistical Physics, edited by K. Binder (Springer, Berlin, 1979).

${ }^{15}$ L. Onsager, Phys. Rev. 65, 117 (1944).

${ }^{16}$ Excellent discussions can be found in B. Widom, in Phase Transitions and Critical Phenomena, edited by C. Domb and M. S. Green (Academic, New York, 1972), Vol. 2, p. 79; J. D. Weeks, J. Chem. Phys. 67, 3106 (1977), and Ref. 5 of this paper. See also J. Bricmont, J. L. Lebowitz, and C. E. Pfister, J. Stat. Phys. 25, 313 (1981).

${ }^{17}$ E. Burkner and D. Stauffer, Z. Phys. B 53, 241 (1983); R. C. Desai and D. Stauffer, J. Phys. A 21, L59 (1988),

18J. S. Langer and L. A. Turski, Acta. Metall. 25, 1113 (1977); K.-t. Leung in Ref.12.

${ }^{19}$ R. Swendesen, Phys. Rev. Phys. B 15, 5421 (1977); J. Adler, ibid. 36, 2473 (1987).

${ }^{20}$ D. B. Abraham, Phys. Rev. Lett. 47, 545 (1981); D. B. Abraham and P. Reed, ibid. 33, 377 (1974); Commun. Math. Phys. 49, 35 (1976).

${ }^{21}$ F. P. Buff, R. A. Lovett, and F. H. Stillinger, Phys. Rev. Lett.
$15,621(1965)$.

${ }^{22}$ J. H. Sikkenk, H. J. Hilhorst, and A. F. Bakker, Physica 131A, 587 (1985).

${ }^{23}$ For a recent review, see M. Barber, in Phase Transitions and Critical Phenomena, edited by C. Domb and J. Lebowitz (Academic, New York, 1984), Vol. 8, p. 145, and references therein.

${ }^{24} \mathrm{See}$, in the case of $X Y$ model and its dual models, Y. Saito and H. Müller-Krumbhaar, Phys. Rev. B 23, 308 (1981); R. Swendsen, ibid. 25, 2019 (1982); W. J. Shugard, J. D. Weeks, and G. H. Gilmer, ibid. 25, 2022 (1982).

${ }^{25}$ See M. S. Wertheim, J. Chem. Phys. 65, 2377 (1976), and Ref. 5 of this paper.

${ }^{26}$ See, e.g., P. C. Hohenberg and B. I. Halperin, Rev. Mod. Phys. 49, 435 (1977).

${ }^{27}$ Theoretically, it is customary to calculate the dispersion relation $\omega(q) \sim q^{z}$. See, e.g., J. S. Langer and L. A. Turski, Ref. 18; G. Jug and D. Jasnow, Phys. Rev. B 31, 7385 (1985); D. Jasnow and R. K. P. Zia, Phys. Rev. A 36, 2243 (1987).

${ }^{28}$ H. Guo and D. Jasnow, Phys. Rev. A 34, 5027 (1986). 